\title{
Grape Seed Polyphenols Ameliorated Dextran Sulfate Sodium-Induced Colitis via Suppression of Inflammation and Apoptosis
}

\author{
Yunxia Wang ${ }^{a-c}$ Yunan Wang ${ }^{a, b}$ Wanli Shen $^{d}$ Yandi Wang $^{a, b}$ Yini Cao $^{a-c}$ \\ Nuerdida Nuerbulatia,b Wen Chen ${ }^{d}$ Guotao Lu ${ }^{e, f}$ Weiming Xiao ${ }^{e, f}$ Rong Qi ${ }^{a-c}$ \\ a Peking University Institute of Cardiovascular Sciences, Peking University Health Science Center, Peking University, \\ Beijing, China; ${ }^{b}$ Key Laboratory of Molecular Cardiovascular Sciences, Ministry of Education, Beijing, China; ${ }^{c}$ Beijing Key \\ Laboratory of Molecular Pharmaceutics and New Drug Delivery Systems, Beijing, China; ${ }^{d}$ School of Pharmacy, Shihezi

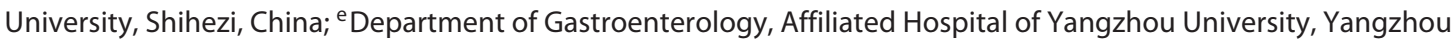 \\ University, Yangzhou, China; fJiangsu Co-Innovation Center for Prevention and Control of Important Animal Infectious \\ Diseases and Zoonoses, College of Veterinary Medicine, Yangzhou University, Yangzhou, China
}

\section{Keywords}

Ulcerative colitis · Grape seed polyphenols · Inflammation · Apoptosis

\begin{abstract}
Background: Ulcerative colitis (UC) is an inflammatory bowel disease. Its onset is typically gradual, usually followed by periods of spontaneous remission and subsequent relapses. Grape seed polyphenols (GSP), a natural product extracted from grape seeds, have strong anti-inflammatory functions. Objectives: In this study, we investigated whether GSP has an inhibitory effect on UC and its related mechanism or not. Methods: We induced UC by $2.5 \%$ dextran sulfate sodium (DSS) and GSP at different doses (500 and $750 \mathrm{mg} / \mathrm{kg}$ body weight per day) was administrated to the mice by gavage. Body weight, diarrhea, and bloody stool were recorded every day to evaluate disease activity index. Hemotoxylin-eosin staining and immunohistochemical staining were used to identify the histological damages and inflammatory infiltration in colon tissues. Real-time polymerase chain reaction was used to evaluate mRNA expression of interleukin (IL)-6,
\end{abstract}

IL-1 $\beta$, and tumor necrosis factor (TNF)- $\alpha$ and the expression of phosphorylated-signal transducer and activator of transcription 3 (STAT3) and STAT3 were assessed by western blot. The immunofluorescent assay was used to evaluate the apoptosis of intestinal epithelial cells (IECS). Results: GSP could alleviate the loss of body weight, diarrhea, bloody stool, the mucosal damage, and inflammatory infiltration. GSP could also downregulate the mRNA expression of inflammatory cytokines IL-6, IL-1 $\beta$, and TNF- $\alpha$ as well as the phosphorylation of STAT3 and ameliorate the apoptosis of IECs. Conclusions: Our study suggests that GSP has protective effects against DSS-induced UC, which may through suppression of inflammation and apoptosis.

(c) 2019 S. Karger AG, Basel

\section{Introduction}

Inflammatory bowel disease (IBD) is a chronic, relapsing, and remitting inflammatory disorder with a multifactorial etiology [1], which is characterized by the presence of extensive areas of ulceration, pronounced infiltra-

\section{KARGER}

(c) 2019 S. Karger AG, Basel

E-Mail karger@karger.com

www.karger.com/pha 
tion of inflammatory cells, and epithelial cell apoptosis in the gut [2]. Because of the lack of effective treatment, the prevalence of IBD becomes relatively high. And its incidence is increasing around the world, making it a great burden for both the individual patients and the society [3].

The main manifestations of IBD include ulcerative colitis (UC) and Crohn's disease, 2 debilitating disorders of the gastrointestinal tract [4]. UC is a complex disease caused by multiple factors, such as immune dysregulation, mucosal barrier dysfunction, and/or a loss of immunological tolerance to commensal microbiota [5], lead to imbalanced and elevated inflammatory cells, aberrant cytokine production, and abnormal apoptosis of intestinal epithelial cells (IECs) [6]. Inflammatory cytokines, such as tumor necrosis factor (TNF)- $\alpha$, interleukin (IL)- $1 \beta$, and IL-6, have been implicated in the pathogenesis of UC $[7,8]$. And the overexpression of inflammatory cytokines was associated with the infiltration of leukocytes, neutrophils, and macrophages in the development of UC [9].

Inflammation could also induce the apoptosis [10]. More and more evidence highlighted the essential roles in the promotion of IECs apoptosis in the injury of colonic tissue and the abnormality of immunology in UC [11]. Plenty of evidence suggested that the number of apoptotic epithelial cells increased with the development of UC, which may cause a change of the epithelial barrier function [12], resulting in pathogenic microorganism infiltration. Consequently, inflammation and IECs apoptosis were potential drivers in the induction and progression of UC. Therefore, the inhibition of inflammation and apoptosis are the main targets to cure UC.

Plant-based remedies are becoming a promising approach for treating various inflammatory disorders because of their strong anti-inflammatory effects [13]. According to a review of several animal models of acute and chronic colitis, when treated with different polyphenols, clinical symptoms of the animal colitis were largely alleviated [14]. Moreover, human trials have shown correlations between polyphenol intake and general benefits to gut health by modulation of inflammatory and oxidative conditions in the bowels [15].

Grape-seed polyphenols (GSP) also have been demonstrated to have significant anti-inflammatory [16] and antiapoptosis [17] effects according to the previous study, which may contribute to the inhibition of IBD. However, there is little research about the effect of GSP on IBD. The present study was intended to investigate the anti-IBD effects of GSP through amelioration of inflammation and apoptosis.

\section{Materials and Methods}

\section{Chemicals}

GSP were kindly provided by Western Animal Husbandry Co., Ltd. (Xinjiang, China). The GSP were extracted from grape seeds with water-ethanol double water phase system. The most abundant phenolic compounds isolated from grape seed are procyanidin, catechins, and picatechin. And content of each polyphenols is about 56.8, 5.2, and 3.6\%.

Dextran sulfate sodium (DSS, molecular weight $36-50 \mathrm{kDa}$ ) was purchased from MP Biomedicals Co., Ltd. (Beijing, China). Antibodies of CD68 were purchased from BOSTER Biological Technology Co., Ltd. (Hubei, China). Rabbit anti-p-signal transducer and activator of transcription 3 (STAT3) and STAT3 primary antibodies were purchased from Cell Signaling Technology Co., Ltd. (MA, USA). Mouse anti-GAPDH primary antibody and horseradish peroxidase-conjugated secondary antibodies were purchased from Santa Cruz (Dallas, TX, USA). Chloral hydrate and all other reagents were purchased from Sigma-Aldrich (Beijing, China).

\section{Ethics}

All procedures involving animals were confirmed to the Regulations for the Administration of Affairs Concerning Experimental Animals published by the State Science and Technology Commission of China and were approved by the Biomedical Ethics Committee of Peking University. And efforts were made to minimize the animal number and reduce suffering throughout the experimental process.

\section{Animal Experiments}

Healthy 8-week-old female C57BL/6 mice weighing 18-20 g were obtained from the Department of Laboratory Animal Science, Peking University Health Science Center (Beijing, China). All mice were housed under the laboratory conditions of 12-h light/dark cycle and temperature $\left(25 \pm 2{ }^{\circ} \mathrm{C}\right)$ and were given free access to food and water. And mouse IBD studies were performed as described previously [18]. The experiment lasted for 6 days. The mice were randomly distributed to Control group, DSS group, DSS with low-dose GSP group (DSS + GSP-L), and DSS with high-dose GSP group (DSS + GSP-H). Mice in the Control group and the DSS group were orally administrated with $200 \mu \mathrm{L}$ of $0.01 \mathrm{~mol} / \mathrm{L}$ phosphate-buffered saline (PBS, $\mathrm{pH}=7.4$ ) once per day; the DSS + GSP-H group and the DSS + GSP-L group received GSP treatment at a dose of 750 and $500 \mathrm{mg} / \mathrm{kg}$ of body weight, respectively, by oral administration once per day and $2.5 \%(\mathrm{w} / \mathrm{v})$ DSS in drinking water from day 1 and continuing till the end of DSS treatment. All animals were fed with a standard chow diet.

\section{Colitis Evaluation}

Mice were monitored daily for body movement, body weight, diarrhea, and bloody stool. Occurrence of bloody diarrhea was evaluated in a clinical way by inspection of anal discharge. Disease activity index (DAI), which was used as one of the assessment criteria to evaluate the severity of UC, was calculated by combining the score of body weight change (0-4), consistency of stool (0-4), and the occurrence of gross blood in the stool (0-4) [19]. Body weight without change or with incremental change was counted as 0 ; the loss of body weight ranging between 1 and $5 \%$ counted as 1 , $5-10 \%$ counted as 2 , and $10-20 \%$ counted as 3 , and the score of 4
10

Pharmacology 2020;105:9-18

DOI: $10.1159 / 000501897$
Wang/Wang/Shen/Wang/Cao/ Nuerbulati/Chen/Lu/Xiao/Qi 
Table 1. Primer sequences used in amplification PCR and semiquantitative real-time polymerase chain reaction

\begin{tabular}{lll}
\hline Primer & Sequence & \\
\hline$\beta$-Actin & $\begin{array}{l}\text { Forward } \\
\text { Reverse }\end{array}$ & $\begin{array}{l}\text { GGCTGTATTCCCCTCCATCG } \\
\text { CCAGTTGGTAACAATGCCATGT }\end{array}$ \\
\hline IL-1 $\beta$ & Forward & TGAATTGGTCATAGCCCGCA \\
& Reverse & GTTGCCCTCAGCAGTAAGGA \\
\hline IL-6 & Forward & CCAAGAGGTGAGTGCTTCCC \\
& Reverse & CTGTTGTTCAGACTCTCTCCCT \\
\hline TNF- $\alpha$ & Forward & CCCTCACACTCACAAACCAC \\
& Reverse & ATAGCAAATCGGCTGACGGT \\
\hline
\end{tabular}

PCR, polymerase chain reaction; IL, interleukin; TNF, tumor necrosis factor.

was given when body weight loss exceeded $20 \%$. For stool consistency and bloody stool, normal feces were counted as 0 , loose stool without blood were counted as 1 , slight loose stool with bleeding stool were counted as 2 , and watery diarrhea with blood was graded 3 and gross bleeding was counted as 4 . The items of subscore were added up to a combined score, which ranged from 0 (no changes) to 8 (extensive body weight loss, diarrhea with gross bloody stool). The combined DAI scores were determined by 2 independent investigators.

\section{Histological Evaluation}

At the end of the experiment period, the mice were sacrificed under anesthesia. The entire colon was excised and placed on an ice plate and cleaned of fat and mesentery. The colon lengths were measured. The distal and proximal colons were taken and fixed in 4\% paraformaldehyde dissolved in $\mathrm{pH} 7.4 \mathrm{PBS}$ and embedded in paraffin for histological analysis. The colon microscopically scored for damage according to the degree of inflammation and the existence of edema and/or ulceration: 0 , normal; 1 , slight inflammation; 2 , slight inflammation and edema; 3 , moderate inflammation and ulceration; and 4, heavy inflammation and ulceration [20]. The histological scores were also determined by 2 independent investigators.

\section{Real-Time Polymerase Chain Reaction Analysis}

RNA was extracted from colon tissues using Trizol Reagent (Molecular Research Center, USA), and cDNA synthesis was performed using the TransScript First-Strand cDNA Synthesis Super Mix (TransGen Biotech). EvaGreen quantitative polymerase chain reaction (PCR) MasterMix (abm, Canada) was used to evaluate mRNA expression levels according to the manufacturer's instructions. Thermal cycling was initiated with an activation step of $30 \mathrm{~s}$ at $95^{\circ} \mathrm{C}$ and followed by 40 cycles of $95^{\circ} \mathrm{C}$ for $5 \mathrm{~s}$ and $60^{\circ} \mathrm{C}$ for $30 \mathrm{~s}$. Right after amplification, melt curve procedures were performed to ensure that primer dimers and other nonspecific products were minimized. The primers used for real-time PCR are shown in Table 1. And transcript levels were normalized to $\beta$-actin, which was used as an endogenous reference.

GSP Inhibited UC

\section{Western Blot Analysis}

Colon tissues were disrupted by homogenization on ice and centrifuged at $4{ }^{\circ} \mathrm{C}(12,000 \mathrm{~g}, 30 \mathrm{~min})$. The supernatants were collected, and protein concentrations were determined with a biscinchonic acid kit (Pierce Biotechnology, Rockford, IL, USA). For western blot, $30 \mu \mathrm{g}$ protein was loaded in each well and resolved by $10 \%$ SDS-PAGE, and the protein bands were then electro-transferred onto a nitrocellulose membrane using the Bio-Rad MiniProtean II apparatus (Bio-Rad Laboratories, Carlsbad, CA, USA). Membranes were blocked in 5\% (w/v) BSA and incubated with anti-rabbit phosphorylated STAT3 $(1: 1,000)$, anti-rabbit STAT3 $(1: 1,000)$, and anti-mouse GAPDH $(1: 5,000)$ primary antibodies at $4{ }^{\circ} \mathrm{C}$ overnight, followed by horseradish peroxidase-conjugated secondary antibodies $(1: 5,000)$ for $1 \mathrm{~h}$ and visualized with enhanced chemiluminescence system (Pierce Biotechnology, Rockford, IL, USA). GAPDH was used as an internal control for data normalization. All bands were quantified by Image J software.

\section{Immunohistochemistry Staining}

For histological analysis, tissue samples were fixed in $4 \%$ paraformaldehyde dissolved in $\mathrm{pH}$ 7.4 PBS and embedded in paraffin and $5 \mu \mathrm{m}$ sections were used for staining. Following removal of paraffin and endogenous peroxidase, tissues were blocked with $5 \%$ sheep serum albumin and then incubated overnight with 100 -fold diluted CD68 primary antibodies at $4{ }^{\circ} \mathrm{C}$. Subsequently, the slides were washed with PBS and then incubated with 500-fold diluted goat anti-rabbit or mice second antibodies conjugated with peroxidase at $37^{\circ} \mathrm{C}$ for $60 \mathrm{~min}$. After counterstained with hematoxylin, the slides were incubated with diaminobenzidine peroxidase substrate to visualize peroxidase activity by light microscopy.

\section{Immunofluorescent Assay}

Terminal deoxynucleotidyl transferase-mediated dUTP nick end-labeling (TUNEL) staining was performed using the in situ Cell Death Detection Kit according to the manufacturer's protocol. Briefly, paraffin-embedded colon tissue sections of $5-\mu \mathrm{m}$ thickness were cut onto glass slides, dewaxed in xylene, hydrated, and treated with proteinase- $\mathrm{K}$. The activity of endogenous peroxidase was blocked when the samples were immersed in $3 \% \mathrm{H}_{2} \mathrm{O}_{2}$ in methanol. TUNEL reaction mixture was then added to the samples. Subsequently, colon tissue sections were rinsed twice with PBS and mounted with cover slips and glycerin. The FITC-labeled TUNEL-positive cells were imaged under a fluorescent microscopy using $488 \mathrm{~nm}$ excitation and $530 \mathrm{~nm}$ emission.

\section{Statistical Analysis}

All data are presented as mean \pm SEM. $p<0.05$ was considered statistically significant in difference. Statistical significance of differences among the groups was analyzed by Student $t$ test. All statistical analyses were performed using GraphPad Prism for Windows (version 4, San Diego, CA, USA).

\section{Results}

\section{GSP Administration Attenuated DSS-Induced Experimental Colitis}

In in vivo study, body weights, diarrhea, and bloody stool were recorded every day. Figure la shows the weight 


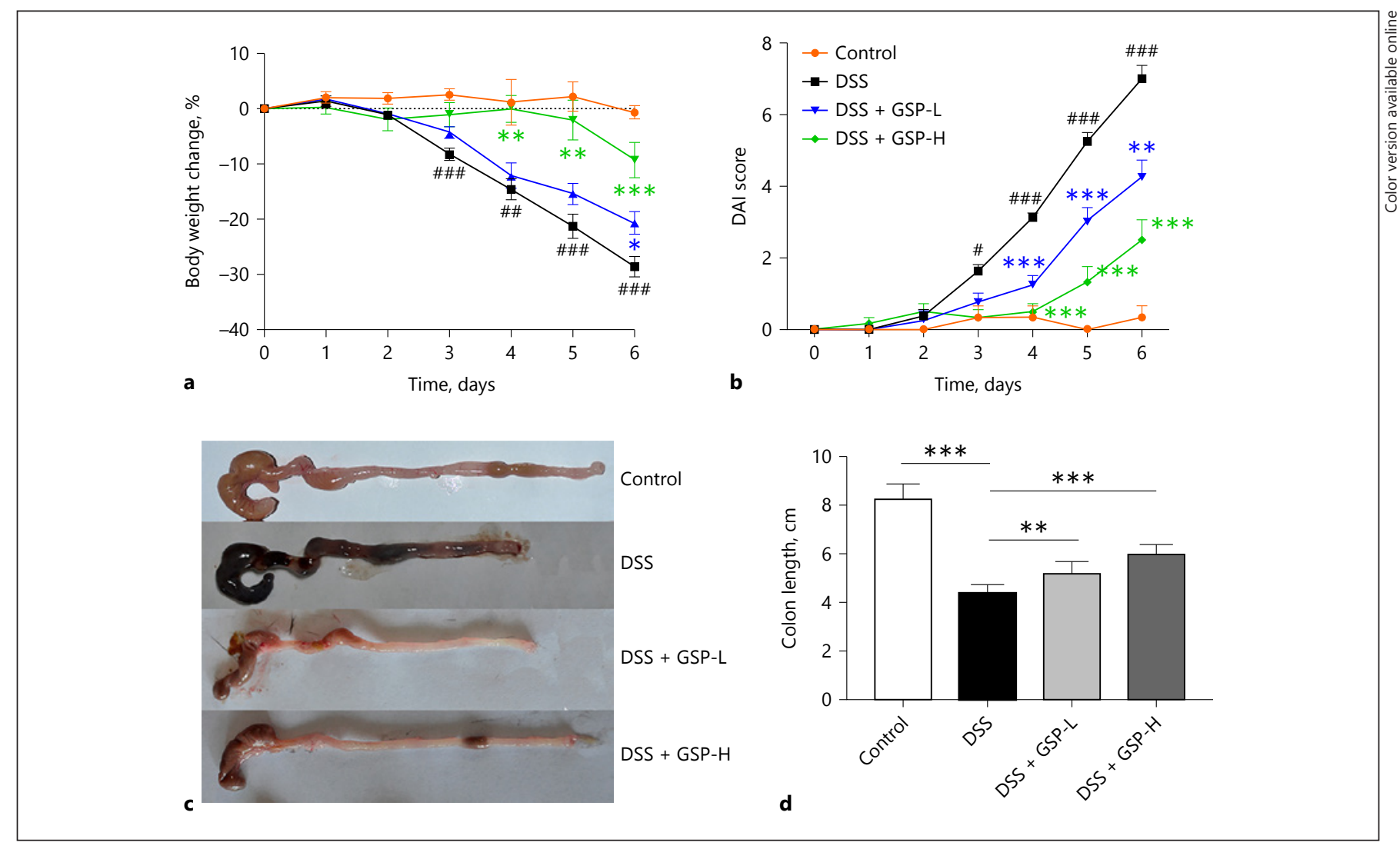

Fig. 1. GSP attenuated the disease progress of DSS-induced UC. a Body weight changes in mice from days 0 to 6 . Data were presented as the percentage of initial body weight. b DAI score, which was the combined score of body weight loss, diarrhea, and bloody stool. c Morphology of colon on day 6. d Colon length of the entire colon. ${ }^{\#} p<0.05,{ }^{\# \#} p<0.01,{ }^{\# \# \#} p<0.001$, compared to the Control group. ${ }^{*} p<0.05,{ }^{* *} p<0.01,{ }^{* * *} p<0.001$, compared to the DSS group. $n=5$ in the Control group and $n=8$ in the other groups. DAI, disease activity index; DSS, dextran sulfate sodium; GSP, grape seed polyphenols. changes of all groups. The body weight of mice in the DSS group distinctly decreased from day 3 . Compared to the DSS group, daily administration of GSP (the DSS + GSP-L group and DSS + GSP-H group), especially at the high dose of $750 \mathrm{mg} / \mathrm{kg}$, effectively attenuated the loss of body weight caused by DSS. Diarrhea symptoms appeared on or shortly after day 3 . From days 3 to 6 , all the mice in the DSS group underwent both severe diarrhea and bloody stool. Mice treated with GSP-H $(750 \mathrm{mg} / \mathrm{kg}$ of body weight) showed normal stool consistency and slight or no visible blood in their feces and crissum, whereas mice treated with the GSP-L (500 mg/kg of body weight) developed slight diarrhea and bloody stool.

We also evaluated the DAI index of all groups according to the aforementioned scoring method. It can be seen from Figure $1 \mathrm{~b}$ that mice in the DSS group achieved the highest score attributed to the prominent body weight loss and serious diarrhea with bloody stool. Mice treated with GSP at both high and low doses showed significantly lower total DAI score compared to that of the DSS group, but with GSP-H better than GSP-L.

Colon shortening is an indirect marker of colonic inflammation [21]. As shown in Figure 1c and d, after drinking DSS water for 6 days, there was significant shortening of the colon length in the DSS mice group compared with those in the Control mice group. Compared to the DSS group, treating the mice with GSP at different doses significantly ameliorated colon shortening. The result indicates that both doses of the GSP showed protective effects on colon shortening, and such effects were in a dose-dependent manner.

\section{GSP Reduced Pathological Damages and}

Inflammatory Infiltration in the Colon

Diagnosis and scoring of colonic tissue samples from each group indicate that GSP protected mouse mucosal
Wang/Wang/Shen/Wang/Cao/ Nuerbulati/Chen/Lu/Xiao/Qi 

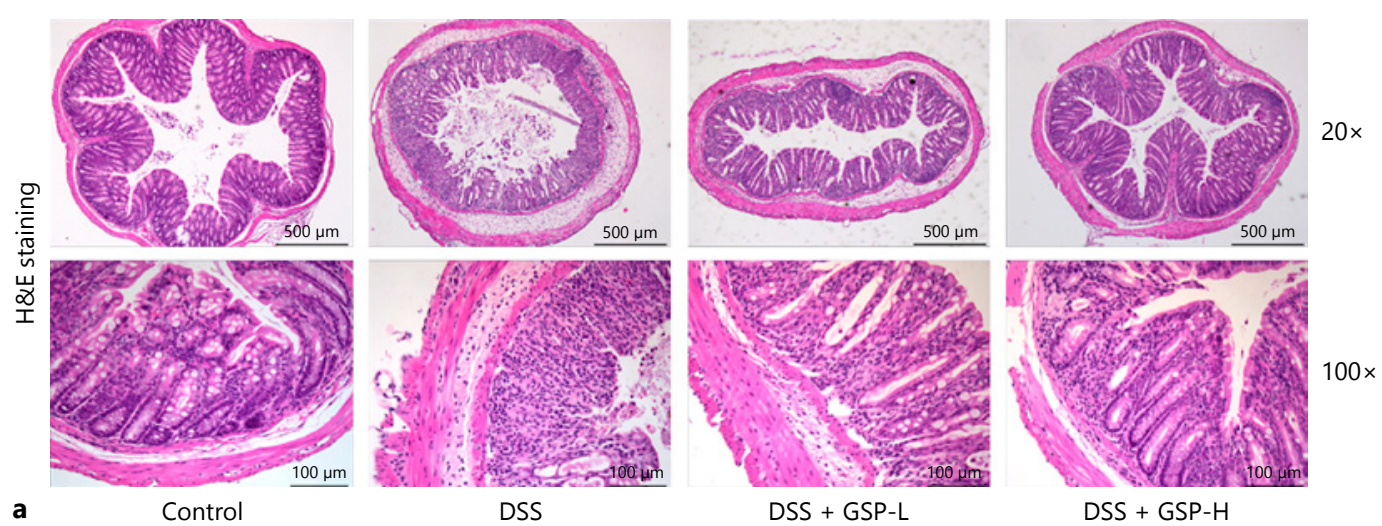

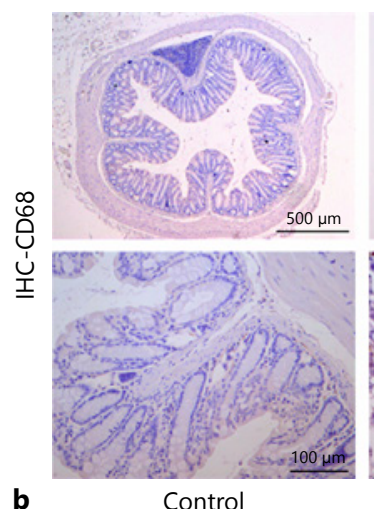

b

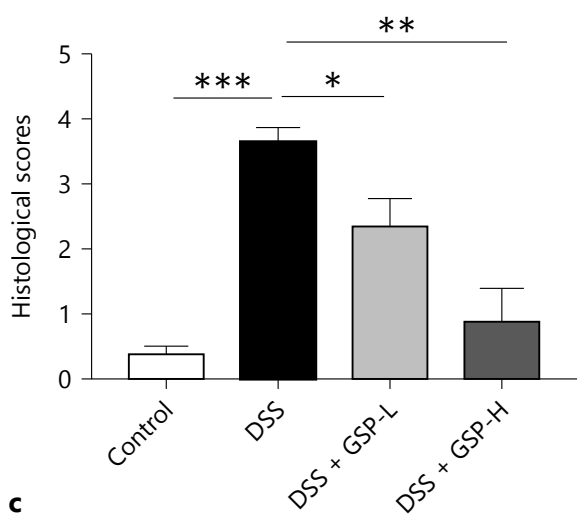

Fig. 2. GSP reduced pathological damages in mucosa and inhibited infiltration of inflammatory cells in the colon of UC mice induced by DSS. a Hemotoxylin and eosin staining at magnification of 20 and $100 \times$. b Immunohistochemical staining of CD68 at magnification of 20 and $100 \times$. c Histological score (the combination of the subscores of mucosal damage and inflammatory cells infiltration). ${ }^{*} p<0.05,{ }^{* *} p<0.01,{ }^{* * *} p<0.001 . n=5$ in the Control group and $n=8$ in the other groups. H\&E, hemotoxylin and eosin; DSS, dextran sulfate sodium; GSP, grape seed polyphenols. epithelium from DSS-induced damage. As shown in Figure $2 \mathrm{a}$ of hemotoxylin and eosin staining and Figure $2 \mathrm{~b}$ of CD68 staining, mucosal epithelium was damaged severely, and there was extensive loss of crypts and goblet cells and great infiltration of inflammatory cells in sub- mucosa in DSS group. In the GSP-treated groups, however, mucosal damage and infiltration of inflammatory cells were ameliorated to a large extent. The result shows that GSP displayed obvious protective effects against these histological damages in the mice colons. 


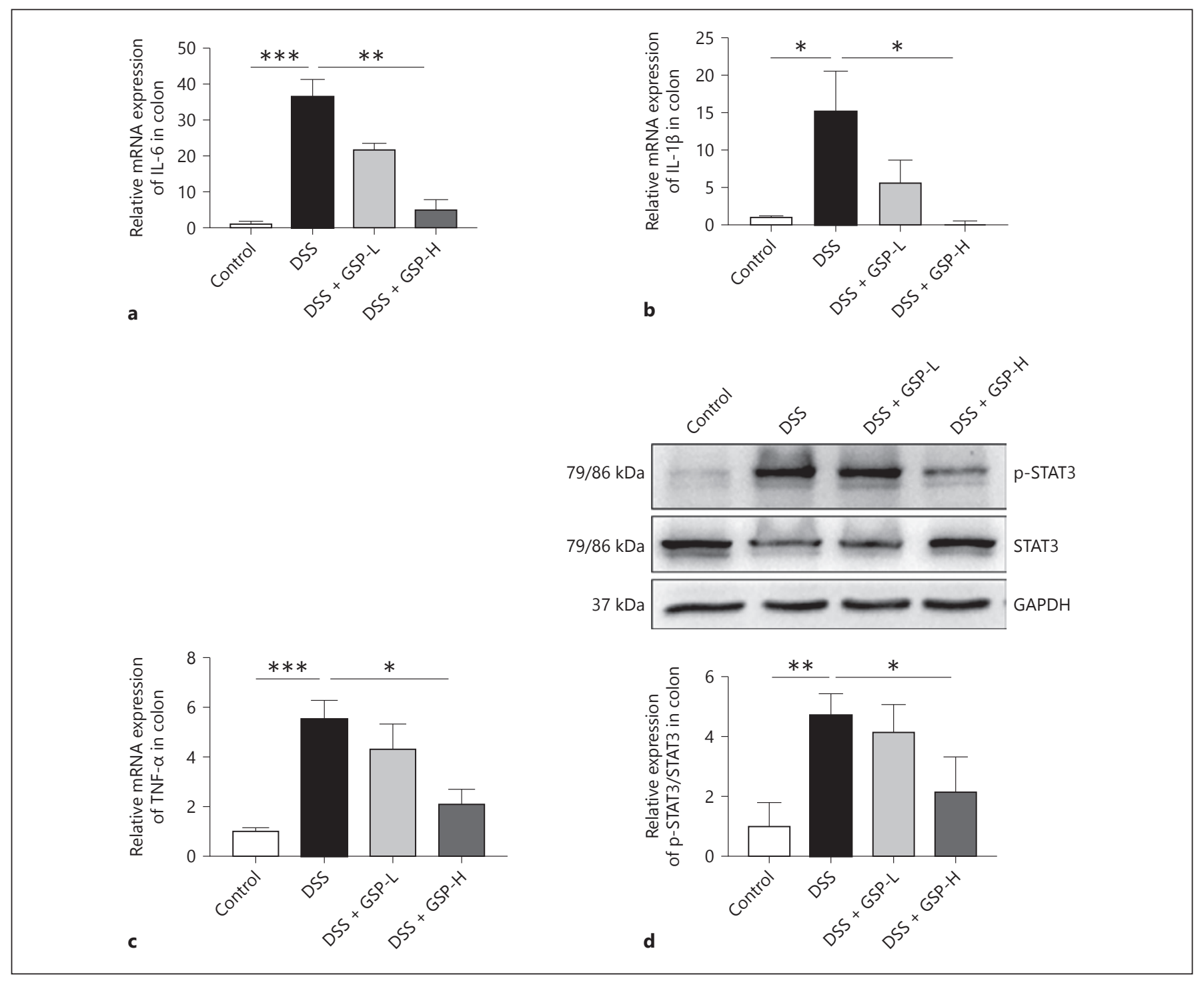

Fig. 3. GSP inhibited mRNA expression of inflammatory cytokines and the phosphorylation of STAT3. a mRNA expression of IL-6. b mRNA expression of IL-1 $\beta$. c mRNA expression of TNF- $\alpha$. d Protein expression level of p-STAT3 and STAT3. Results are ex-

Histological score was evaluated according to the aforementioned scoring method. It can be seen from Figure $2 \mathrm{c}$ that consistent with the DAI score.

GSP-Inhibited mRNA Expression of Proinflammatory Mediators and Phosphorylation/Activation of STAT in the Colon

To further determine the impact of GSP on DSS-induced colitis, quantitative real-time PCR analyses of several proinflammatory mediator genes were investigated. The results showed that mRNA expression of IL-6, IL-1 $\beta$, pressed as mean \pm SEM in each group. ${ }^{*} p<0.05,{ }^{* *} p<0.01,{ }^{* * *} p<$ 0.001. $n=5$ in each group. IL, interleukin; TNF, tumor necrosis factor; DSS, dextran sulfate sodium; GSP, grape seed polyphenols; STAT3, signal transducer and activator of transcription 3.

and TNF- $\alpha$ was remarkably induced in inflamed colons of the mice exposed to DSS and GSP treatment downregulated inflammatory genes (Fig. $3 \mathrm{a}-\mathrm{c}$ ). The results indicate that amelioration of GSP- to DSS-induced colitis was related to its suppression of inflammation.

The activation of STAT pathway has been implicated in the pathogenesis of IBD. Therefore, we assessed the effects of GSP on the activation of STAT signaling pathway. As shown in Figure 3d, the phosphorylation (activation) of STAT3 was significantly induced in the colon mucosa after 6 days of DSS treatment compared with the normal 


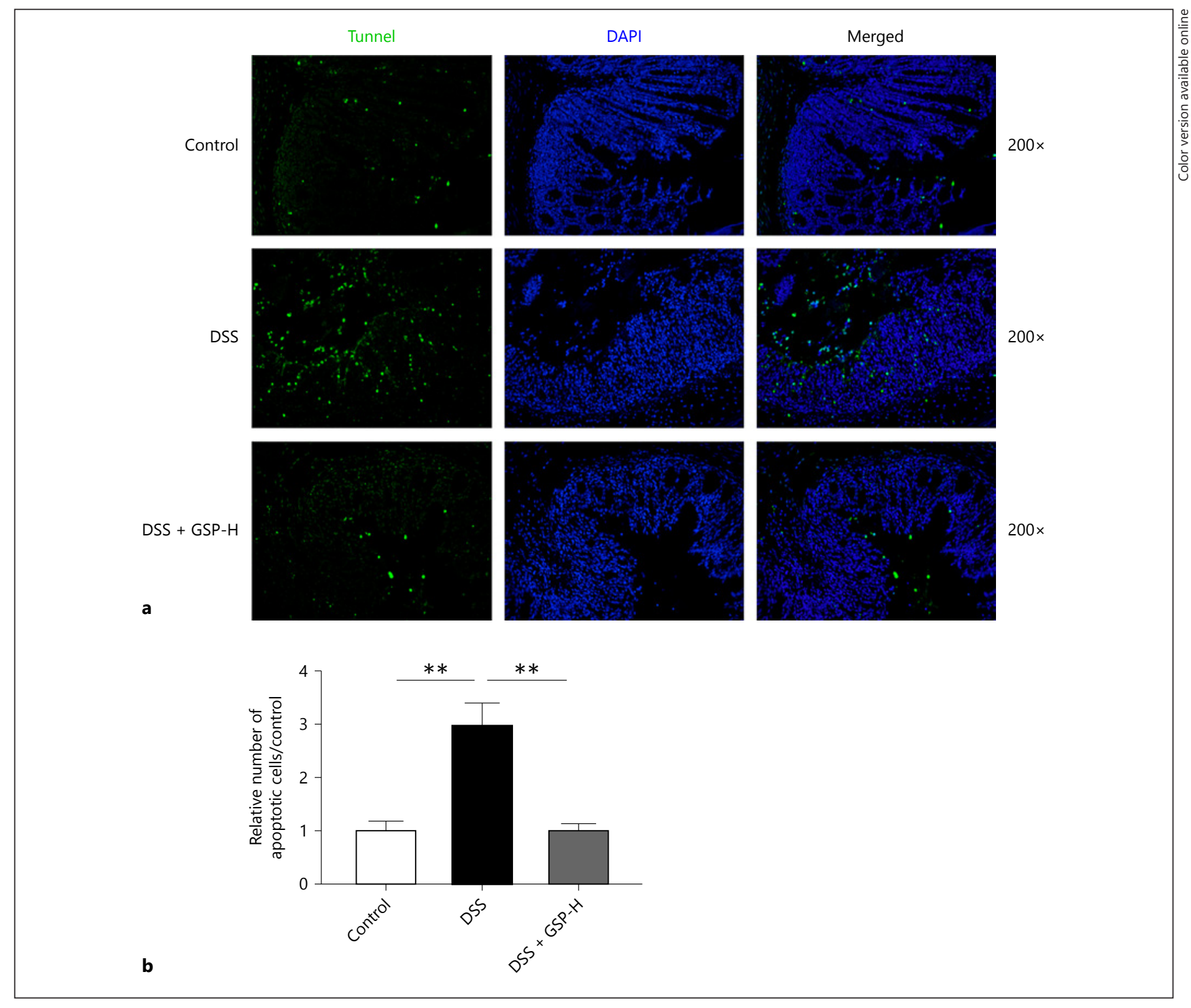

Fig. 4. Effect of GSP on IEC apoptosis. TUNEL staining in colon tissues (a) and quantification (b). The images of TUNEL positive cells were captured by a fluorescence microscope $(200 \times)$. ${ }^{* *} p<0.01, n=4$ in each group. DSS, dextran sulfate sodium; GSP, grape seed polyphenols; DAPI, 4',6-diamidino-2-phenylindole.

Control group. Comparatively, treatment with GSP-H pronouncedly inhibited the increased phosphorylation of STAT3 in the mice colon tissues induced by DSS.

\section{Effect of GSP on IEC Apoptosis}

To study whether experimental colitis was associated with IEC apoptosis and the effect of GSP on IEC apoptosis, we employed TUNEL staining in colonic tissues. As shown in Figure 4, few apoptotic cells were observed in the control group whereas colon tissues presented a marked appearance of apoptotic cells after treatment with DSS. In contrast, treatment with GSP remarkably reduced the percentages of TUNEL-positive cells.

\section{Discussion}

IBD is a very tough health-care problem, and its global incidence has been increasing [3]. The basic characterization of IBD is the chronic-relapsing inflammation in the 
intestines, and clinical trials indicate that the risk of developing colorectal cancer was high in patients with UC [22]. Although significant effort has been dedicated to the treatment of UC in patients, the effects of these treatment regimens in curing UC and preventing colitis-associated colorectal cancer remain disappointing [23]. Therefore, it's urgent to find an effective and safe treatment for UC.

The trend of living a healthy life has led to the concept that plant-based diet is healthier than a diet laden with meat. GSP is a group of active natural polyphenols extracted from dietary plants, and it has attracted much attention because of their remarkable anti-inflammatory effects $[24,25]$. Some literature reported that polyphenols of grape seeds may inhibit enzymes associated with inflammatory reactions. The main constituent in grape seeds, procyanidin, can inhibit the synthesis and release of inflammatory cytokines, such as leukotrienes [26, 27]. And Vennat et al. [28] reported that procyanidin could inhibit gastric ulcer. Other researchers also have found the antiulcer activity of grape seed extracts [29]. In this study, we confirmed the protective effects of GSP on IBD.

From the third day of DSS administration, mice began to show reduced activity, loose stool, and decreased body weight. During later phases, mice had serious bloody stool, and a few mice died. Hyperemia and edema of the colon and bloody contents in the colon were also observed. The intestinal tract wall became thick and stiff. Shortening of the colon was found in all DSS-treated mice. Moreover, histopathological examination showed that the crypt structure in the colon tissue disappeared and epithelial and goblet cells decreased, while inflammatory cell infiltrated into the mucosa and submucosa. However, after GSP treatment, the lesion severity decreased, the extent of colon injury reduced, and the infiltration of inflammatory cells alleviated. These results confirm that GSP had inhibitory effects on UC.

The mechanisms involved in the onset and progression of IBD are very complex. In addition to genetic and environmental factors as well as mucosal immune system of the intestinal tract, the imbalance between proinflammatory and anti-inflammatory cytokines plays a vital role in the progress of IBD [30]. In chronic mucosal inflammation during IBD, macrophages can be activated and produce a large quantity of proinflammatory cytokines, such as IL- 6 and IL- $\beta$ [31]. In addition, in UC patients, the mRNA expression levels of several cytokines, including interferon- $\gamma$, TNF- $\alpha$, IL- $1 \beta$, and IL- 6 , increased and were positively associated with the severity of UC [32]. Among these cytokines, overexpression of IL- 6 and IL- $1 \beta$ is critical in intestinal mucosal lesions. These inflamma- tory cytokines could also induce the infiltration of leukocytes, neutrophils, and macrophages. In our study, we found that treatment with GSP decreased the mRNA expression of IL-6, IL- $1 \beta$, and TNF- $\alpha$ as well as the infiltration of CD68-positive macrophages. Therefore, we speculated that GSP reduced the severity of colitis lesions by inhibiting the increased inflammatory response.

STAT3 plays an important role in various disorders, including IBD [33]. Various cytokines and growth factors can induce the activation of STAT3 [34]. The activation of STAT3 was also related to the inflammatory status of colons in UC; more specifically, STAT3 activation probably participates in promoting inflammation by enhancing the production of inflammatory cytokines. Therefore, suppressing the activity of STAT3 may be necessary for reducing inflammation in the colon [32]. In our study, we found that GSP reduced the phosphorylation of STAT3, which terminated further activation of STAT3, and finally contributed to the healing of colitis.

Epithelial barrier, which can protect against the damage caused by antigens such as small ions and larger molecules, has been shown to be seriously impaired in IBD [35]. The elevated proinflammatory cytokines in UC patients with UC were considered to play a central role in the defect of the epithelial barrier. Moreover, the disturbance in the epithelial barrier function may further cause intestinal inflammation [36, 37]. Our study found that GSP could attenuate inflammation, and previous studies suggested that grape seed extracts exert protective effects to the ileal epithelial structure and barrier function through suppressing inflammatory response $[38,39]$.

Apoptosis in the intestinal epithelium has been considered one of the major hallmarks of IBD [40]. In UC, inflammation can induce apoptosis of epithelial cells, leading to the disruption of the mucosal integrity and the subsequent change in the function of the epithelial barrier, and finally the invasion bacterial pathogens $[12,41]$. In the present study, we found that GSP could inhibit the apoptosis of the colonic epithelium. In addition, there were less TUNEL-positive colonic epithelial cells in the GSP-treated mice compared to those in the DSS positive control mice, indicating that GSP could ameliorate injury of epithelial barriers.

In conclusion, this study indicated that GSP ameliorated DSS-induced colitis, which was probably through downregulation of the inflammation and apoptosis. Oral administration of GSP can be a potential remedy for preventing the occurrence of IBD. The specific compositions and active monomers in GSP will be identified in our future studies.
Wang/Wang/Shen/Wang/Cao/ Nuerbulati/Chen/Lu/Xiao/Qi 


\section{Statement of Ethics}

All procedures involving animals were confirmed to the Regulations for the Administration of Affairs Concerning Experimental Animals published by the State Science and Technology Commission of China and were approved by the Biomedical Ethics Committee of Peking University. And efforts were made to minimize the animal number and reduce suffering throughout the experimental process.

\section{Disclosure Statement}

The authors declare that they have no conflict of interest.

\section{Funding Sources}

This work was supported by the National Natural Science Foundation of China (No. 81770268, 81360054) and the National Basic Research Program of China (No. 2015CB932100). We gratefully acknowledge all of the funding sources.

\section{Author Contributions}

All authors contributed extensively to the work presented in this paper. R.Q., W.C., Yunxia Wang, and Yunan Wang: designed the experiments. R.Q. and Yunxia Wang: wrote up the manuscript and prepared figures. Yunxia Wang, Yunan Wang, and N.N.: executed the experiments. Yandi Wang, Y.C., W.S., G.L., and W.X. provided help with the laboratory technique and experiments.

\section{References}

1 Hartog A, Belle FN, Bastiaans J, de Graaff P, Garssen J, Harthoorn LF, et al. A potential role for regulatory $\mathrm{T}$-cells in the amelioration of DSS induced colitis by dietary non-digestible polysaccharides. J Nutr Biochem. 2015 Mar;26(3):227-33.

2 Dou W, Zhang J, Ren G, Ding L, Sun A, Deng $\mathrm{C}$, et al. Mangiferin attenuates the symptoms of dextran sulfate sodium-induced colitis in mice via NF- $\mathrm{KB}$ and MAPK signaling inactivation. Int Immunopharmacol. 2014 Nov; 23(1):170-8.

3 Xavier RJ, Podolsky DK. Unravelling the pathogenesis of inflammatory bowel disease. Nature. 2007 Jul;448(7152):427-34.

4 de Lange KM, Barrett JC. Understanding inflammatory bowel disease via immunogenetics. J Autoimmun. 2015 Nov;64:91-100.

5 Hiller F, Besselt K, Deubel S, Brigelius-Flohé R, Kipp AP. GPx2 Induction Is Mediated Through STAT Transcription Factors During Acute Colitis. Inflamm Bowel Dis. 2015 Sep; 21(9):2078-89.

6 Dou W, Zhang J, Sun A, Zhang E, Ding L, Mukherjee $S$, et al. Protective effect of naringenin against experimental colitis via suppression of Toll-like receptor $4 / \mathrm{NF}-\kappa B$ signalling. Br J Nutr. 2013 Aug;110(4):599-608.

7 Papadakis KA, Targan SR. Role of cytokines in the pathogenesis of inflammatory bowel disease. Annu Rev Med. 2000;51(1):289_ 98.

8 Gunning JE, Tomasulo JP, Garite T. Laparoscopic tubal sterilization using thermal coagulation. Obstet Gynecol. 1979 Oct;54(4):5059.

9 Mantovani A, Muzio M, Garlanda C, Sozzani S, Allavena P. Macrophage control of inflammation: negative pathways of regulation of inflammatory cytokines. Novartis Found Symp. 2001;234:120-31.

10 Edelblum KL, Yan F, Yamaoka T, Polk DB. Regulation of apoptosis during homeostasis and disease in the intestinal epithelium. Inflamm Bowel Dis. 2006 May;12(5):413-24.

11 Iwamoto M, Koji T, Makiyama K, Kobayashi N, Nakane PK. Apoptosis of crypt epithelial cells in ulcerative colitis. J Pathol. 1996 Oct; 180(2):152-9.

12 Qiu W, Wu B, Wang X, Buchanan ME, Regueiro MD, Hartman DJ, et al. PUMA-mediated intestinal epithelial apoptosis contributes to ulcerative colitis in humans and mice. J Clin Invest. 2011 May;121(5):1722-32.

13 Heber D, Zhang Y, Yang J, Ma JE, Henning SM, Li Z. Green tea, black tea, and oolong tea polyphenols reduce visceral fat and inflammation in mice fed high-fat, high-sucrose obesogenic diets. J Nutr. 2014 Sep;144(9): 1385-93.

14 Martin DA, Bolling BW. A review of the efficacy of dietary polyphenols in experimental models of inflammatory bowel diseases. Food Funct. 2015 Jun;6(6):1773-86.

15 Dryden GW, Lam A, Beatty K, Qazzaz HH, McClain CJ. A pilot study to evaluate the safety and efficacy of an oral dose of (-)-epigallocatechin-3-gallate-rich polyphenon $\mathrm{E}$ in patients with mild to moderate ulcerative colitis. Inflamm Bowel Dis. 2013 Aug;19(9):1904-12.

16 Shi J, Yu J, Pohorly JE, Kakuda Y. Polyphenolics in grape seeds-biochemistry and functionality. J Med Food. 2003;6(4):291-9.

17 Zhang Z, Zheng L, Zhao Z, Shi J, Wang X, Huang J. Grape seed proanthocyanidins inhibit H2O2-induced osteoblastic MC3T3-E1 cell apoptosis via ameliorating $\mathrm{H} 2 \mathrm{O} 2$-induced mitochondrial dysfunction. J Toxicol Sci. 2014;39(5):803-13.

18 Dou W, Mukherjee S, Li H, Venkatesh M, Wang $\mathrm{H}$, Kortagere $\mathrm{S}$, et al. Alleviation of gut inflammation by $\mathrm{Cdx} 2 / \mathrm{Pxr}$ pathway in a mouse model of chemical colitis. PLoS One. 2012;7(7):e36075.

19 Cooper HS, Murthy SN, Shah RS, Sedergran DJ. Clinicopathologic study of dextran sulfate sodium experimental murine colitis. Lab Invest. 1993 Aug;69(2):238-49.

20 Zhang CL, Zhang S, He WX, Lu JL, Xu YJ, Yang $\mathrm{JY}$, et al. Baicalin may alleviate inflammatory infiltration in dextran sodium sulfate-induced chronic ulcerative colitis via inhibiting IL-33 expression. Life Sci. 2017 Oct; 186:125-32.

21 Okayasu I, Hatakeyama S, Yamada M, Ohkusa T, Inagaki Y, Nakaya R. A novel method in the induction of reliable experimental acute and chronic ulcerative colitis in mice. Gastroenterology. 1990 Mar;98(3):694-702.

22 Dyson JK, Rutter MD. Colorectal cancer in inflammatory bowel disease: what is the real magnitude of the risk. World J Gastroenterol. 2012 Aug; 18(29):3839-48.

23 Rutter MD, Saunders BP, Wilkinson KH, Rumbles S, Schofield G, Kamm MA, et al. Thirty-year analysis of a colonoscopic surveillance program for neoplasia in ulcerative colitis. Gastroenterology. 2006 Apr;130(4):1030-8.

24 Rodríguez-Ramiro I, Ramos S, López-Oliva E, Agis-Torres A, Bravo L, Goya L, et al. Cocoa polyphenols prevent inflammation in the colon of azoxymethane-treated rats and in TNFa-stimulated Caco- 2 cells. Br J Nutr. 2013 Jul; 110(2):206-15

25 Cheng A, Yan H, Han C, Wang W, Tian Y, Chen X. Polyphenols from blueberries modulate inflammation cytokines in LPS-induced RAW264.7 macrophages. Int J Biol Macromol. 2014 Aug;69:382-7.

26 Martinez-Micaelo N, González-Abuín N, Ardèvol A, Pinent M, Blay MT. Procyanidins and inflammation: molecular targets and health implications. Biofactors. 2012 Jul-Aug; 38(4):257-65.

27 Bitzer ZT, Glisan SL, Dorenkott MR, Goodrich KM, Ye L, O'Keefe SF, et al. Cocoa procyanidins with different degrees of polymerization possess distinct activities in models of colonic inflammation. J Nutr Biochem. 2015 Aug;26(8):827-31. 
28 Vennat B, Gross D, Pourrat H, Pourrat A, Bastide P, Bastide J. Anti-ulcer activity of procyanidins preparation of water-soluble procyanidin-cimetidine complexes. Pharm Acta Helv. 1989;64(11):316-20.

29 Cheah KY, Bastian SE, Acott TM, Abimosleh SM, Lymn KA, Howarth GS. Grape seed extract reduces the severity of selected disease markers in the proximal colon of dextran sulphate sodium-induced colitis in rats. Dig Dis Sci. 2013 Apr;58(4):970-7.

30 Moldoveanu AC, Diculescu M, Braticevici CF. Cytokines in inflammatory bowel disease. Rom J Intern Med. 2015 Apr-Jun;53(2):118-27.

31 Rogler G, Andus T. Cytokines in inflammatory bowel disease. World J Surg. 1998 Apr; 22(4):382-9.

32 Nguyen AV, Wu YY, Lin EY. STAT3 and sphingosine-1-phosphate in inflammationassociated colorectal cancer. World J Gastroenterol. 2014 Aug;20(30):10279-87.
33 Lee SY, Lee SH, Yang EJ, Kim EK, Kim JK, Shin DY, et al. Metformin Ameliorates Inflammatory Bowel Disease by Suppression of the STAT3 Signaling Pathway and Regulation of the between Th17/Treg Balance. PLoS One. 2015 Sep;10(9):e0135858.

34 Nguyen PM, Putoczki TL, Ernst M. STAT3Activating Cytokines: A Therapeutic Opportunity for Inflammatory Bowel Disease. J Interferon Cytokine Res. 2015 May;35(5):340-50.

35 Bjarnason I, MacPherson A, Hollander D. Intestinal permeability: an overview. Gastroenterology. 1995 May;108(5):1566-81.

36 Schmitz H, Fromm M, Bentzel CJ, Scholz P, Detjen K, Mankertz J, et al. Tumor necrosis factor-alpha (TNFalpha) regulates the epithelial barrier in the human intestinal cell line HT29/B6. J Cell Sci. 1999 Jan;112(Pt 1):137-46.

37 Bürgel N, Bojarski C, Mankertz J, Zeitz M, Fromm M, Schulzke JD. Mechanisms of diarrhea in collagenous colitis. Gastroenterology. 2002 Aug;123(2):433-43.
38 Yang G, Wang H, Kang Y, Zhu MJ. Grape seed extract improves epithelial structure and suppresses inflammation in ileum of IL10-deficient mice. Food Funct. 2014 Oct; 5(10):2558-63.

39 Yang G, Xue Y, Zhang H, Du M, Zhu MJ. Favourable effects of grape seed extract on intestinal epithelial differentiation and barrier function in IL10-deficient mice. Br J Nutr. 2015 Jul;114(1):15-23.

40 Nunes T, Bernardazzi C, de Souza HS. Cell death and inflammatory bowel diseases: apoptosis, necrosis, and autophagy in the intestinal epithelium. BioMed Res Int. 2014; 2014:218493

41 Zeissig S, Bojarski C, Buergel N, Mankertz J, Zeitz M, Fromm M, et al. Downregulation of epithelial apoptosis and barrier repair in active Crohn's disease by tumour necrosis factor alpha antibody treatment. Gut. 2004 Sep; 53(9):1295-302. 